\title{
Integrated Microstrip Lines With Co-Ta-Zr Magnetic Films
}

\author{
Pedram Khalili Amiri ${ }^{1}$, Behzad Rejaei ${ }^{1}$, Yan Zhuang ${ }^{2}$, Marina Vroubel ${ }^{3}$, Dok Won Lee ${ }^{4}$, Shan X. Wang ${ }^{4}$, and \\ Joachim N. Burghartz ${ }^{5}$, Fellow, IEEE \\ ${ }^{1}$ Delft Institute of Microsystems and Nanoelectronics (DIMES), Delft University of Technology, \\ Delft NL-2628CD, The Netherlands \\ ${ }^{2}$ Department of Electrical Engineering, Wright State University, Dayton, OH 45435-0001 USA \\ ${ }^{3}$ NXP Semiconductors, Nijmegen, NL-6534AE, The Netherlands \\ ${ }^{4}$ Department of Materials Science and Engineering, Stanford University, Stanford, CA 94305-4045 USA \\ ${ }^{5}$ Institute for Microelectronics Stuttgart (IMS CHIPS), Stuttgart 70569, Germany
}

\begin{abstract}
Microstrip transmission lines with ferromagnetic Co-Ta-Zr cores are investigated in this paper. Compared to nonmagnetic devices, an increase in both inductance $(\times 11)$ and quality factor $(\times 6)$ is achieved in the transmission lines. The role of the magnetic material's electrical resistivity and the effect of the shape-induced anisotropy field (created by patterning the magnetic film into narrow stripes) are discussed. It is shown that the nonuniform distribution of the shape anisotropy inside the Co-Ta-Zr pattern can be used to advantage by proper placement of the signal line, thereby increasing the ferromagnetic resonance (FMR) frequency of the magnetic core. Inductance enhancement is achieved at frequencies up to $\sim 10 \mathrm{GHz}$.
\end{abstract}

Index Terms-Magnetic anisotropy, magnetic microwave devices, microstrip, planar transmission lines, soft magnetic films.

\section{INTRODUCTION}

I NTEGRATED passive devices such as transmission lines and inductors are indispensable components of contemporary radio frequency $(\mathrm{RF})$ integrated circuits (ICs). They find applications in filters, impedance matching networks, resonators, couplers, and various other on-chip elements. These RF passives, however, typically suffer from small inductance per unit area and high loss (quantified by a low quality factor $Q$ ).

In order to overcome these limitations, monolithic integration of IC-compatible metallic ferromagnetic films has been increasingly studied in recent years [1]-[19]. Performance enhancement and size reduction have already been demonstrated in on-chip RF inductors [1]-[6] and transmission line elements [7]-[10]. Moreover, enhanced functionalities such as tunable [11]-[13] and nonreciprocal [14], [15] devices have been shown to be possible using silicon-compatible magnetic thin films.

Nevertheless, the high-energy dissipation brought about by parasitic eddy currents in the metallic ferromagnetic layers and the limited frequency range of operation due to ferromagnetic resonance (FMR) have thus far limited the performance of integrated magnetic RF devices, impeding their application in a practical setting. For example, while the use of thick magnetic films can bring about a significant increase in inductance, measurements on microstrip transmission lines with $\mathrm{Ni}-\mathrm{Fe}$ cores show that an increase of magnetic film thickness from 200 to $500 \mathrm{~nm}$ reduces the $Q$ factor by a factor of $\sim 2$ due to enhanced eddy current loss, essentially rendering thicker Ni-Fe films useless [8]-[10]. Moreover, for typical Ni-Fe layers, FMR limits the useful operation frequency range to below $\sim 2 \mathrm{GHz}$ [10]. While the application of an external dc magnetic field during device operation can increase the FMR frequency [7], this option is undesirable in on-chip applications for practical reasons.

Digital Object Identifier 10.1109/TMAG.2008.2002432
As a result, improved silicon-compatible magnetic materials with higher resistivity (to suppress the flow of eddy currents) and increased FMR frequency (i.e., large anisotropy field and saturation magnetization) are highly called for in order to better realize the potential benefits of integrated magnetic RF components.

This paper presents experiments on microstrip transmission lines based on such a candidate material. The magnetic film consists of amorphous $\mathrm{Co}-\mathrm{Ta}-\mathrm{Zr}$ with a relatively high resistivity [5], [17], which translates into a large skin depth when used as a magnetic core for on-chip passives. The latter allows for the integration of a relatively thick $(1 \mu \mathrm{m})$ magnetic film, leading to an order of magnitude increase in the microstrip inductance per unit length when compared to nonmagnetic devices. Moreover, a combination of internal and shape-induced anisotropy fields leads to a fairly high FMR frequency, allowing for the inductance enhancement to be maintained up to frequencies as high as $10 \mathrm{GHz}$.

\section{EXPERIMENT AND DISCUSSION}

Ferromagnetic amorphous $\mathrm{Co}-\mathrm{Ta}-\mathrm{Zr}$ films with a resistivity of $100 \mu \Omega-\mathrm{cm}(\sim 6$ times higher than Ni-Fe) and saturation magnetization of $\sim 1.3 \mathrm{~T}$ were deposited by RF diode sputtering. The $1-\mu \mathrm{m}$-thick magnetic films were then patterned into $100-\mu \mathrm{m}$-wide, 1 -mm-long stripes and incorporated into microstrip transmission lines on a standard silicon wafer. A schematic of the structure is shown in Fig. 1. The ground and signal lines were sputtered $\mathrm{Al}$ layers with thicknesses of $2 \mu \mathrm{m}$ and $3 \mu \mathrm{m}$, respectively, and the ground plane was separated from the underlying silicon substrate by a $2-\mu$ m-thick thermal oxide layer. An internal anisotropy field of $\sim 20$ Oe was induced in the $\mathrm{Co}-\mathrm{Ta}-\mathrm{Zr}$ layers by applying a magnetic field during sputter deposition, as can be seen from B-H loop measurements shown in Fig. 2.

In addition, demagnetizing fields arising from the lateral confinement of the magnetic stripe lead to a so-called shape-induced anisotropy field [18], [19]. The latter adds up with the 
(a)

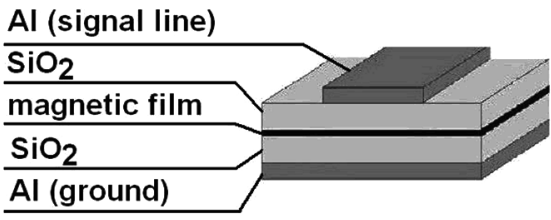

(b)

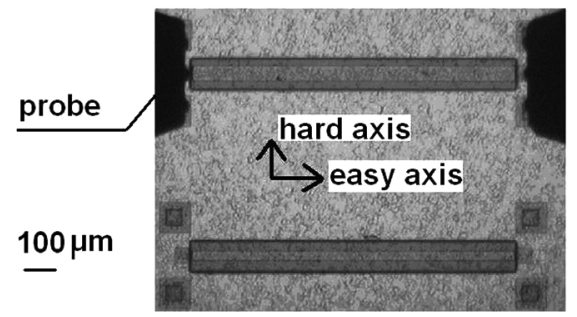

Fig. 1. Schematic representation (a) and top view microphotograph (b) of microstrip transmission lines with magnetic cores. The Co-Ta-Zr magnetic film was $1-\mu \mathrm{m}$-thick in our experiments, with a saturation magnetization of $\sim 1.3 \mathrm{~T}$ and a coercivity of $<1 \mathrm{Oe}$. The sputtered $\mathrm{Al}$ ground and signal line thicknesses were $2 \mu \mathrm{m}$ and $3 \mu \mathrm{m}$, respectively. The magnetic core was patterned into rectangular stripes with dimensions of $1 \mathrm{~mm} \times 100 \mu \mathrm{m}$.

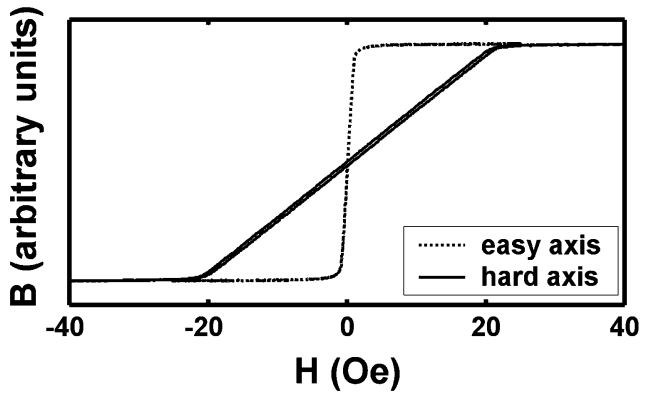

Fig. 2. B-H loop measurements along the hard and easy axes of the (unpatterned) $\mathrm{Co}-\mathrm{Ta}-\mathrm{Zr}$ film, indicating an internal magnetic anisotropy field of $\sim 20$ Oe. Magnetic properties of the Co-Ta-Zr layer and spiral inductors based on this material are further discussed in [5], [17].

internal anisotropy induced during deposition, resulting in an increase of the FMR frequency. The shape anisotropy is nonuniform over the stripe width, its value sharply increasing towards the edges of the magnetic pattern, where the demagnetizing fields are larger [20]-[22]. For this reason, one can expect devices to exhibit higher FMR frequencies if their signal lines are placed near the edges of the magnetic core rather than on top of its center region. Both types of devices were fabricated in our experiment in order to facilitate a comparison, as described below.

The microstrip lines were characterized by scattering parameter measurements on a line-reflect-match-calibrated HP-8510 network analyzer in connection with a Cascade Microtech probe station. A ground-signal-ground (GSG) two-port configuration was used for the measurements, and no external magnetic field was applied to the devices. The results were then transformed to impedance parameters, from which the inductance $L$, resistance $R$, and capacitance $C$ (per unit length) were obtained.

The inductance and quality factor of microstrip lines with and without a $\mathrm{Co}-\mathrm{Ta}-\mathrm{Zr}$ magnetic core are compared in Fig. 3. The signal line is $50 \mu \mathrm{m}$ wide in this case and is located near the

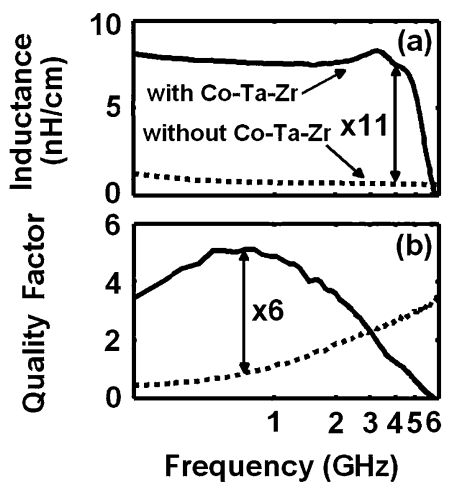

Fig. 3. (a) Inductance per unit length and (b) quality factor enhancement in microstrip transmission lines with a magnetic $\mathrm{Co}-\mathrm{Ta}-\mathrm{Zr}$ core. The signal line width is $50 \mu \mathrm{m}$ in this case.

center of the magnetic stripe, as depicted in Fig. 1. The quality factor is given by $Q=\beta / 2 \alpha$, where $\alpha$ and $\beta$ are the real and imaginary parts of the complex propagation constant $\gamma=\alpha+$ $j \beta=\sqrt{j \omega C(R+j \omega L)}$. Here, we have neglected the shunt conductance per unit length $G$, which is small for the dielectric layers used in our devices. The microstrips show an increase of inductance by a factor of $\sim 11$ at $4 \mathrm{GHz}$. Moreover, $L$ remains constant up to $\sim 4.5 \mathrm{GHz}$, and is higher than the inductance of the control lines up to $\sim 6 \mathrm{GHz}$. The $Q$ factor increases by a factor of $\sim 6$ at $0.7 \mathrm{GHz}$ and remains higher than that of the control lines up to $\sim 3 \mathrm{GHz}$.

Note that the fairly high FMR frequency in this device is mostly a result of the shape-induced magnetic anisotropy field, as the 20 Oe internal anisotropy is too small to lead to such a resonance value. The high resistivity (large skin depth) of the $\mathrm{Co}-\mathrm{Ta}-\mathrm{Zr}$ material thus allows for the incorporation of a relatively thick $(1 \mu \mathrm{m})$ magnetic film without inducing excessive conductive loss, thus maintaining a reasonable enhancement of $Q$ factor in the device. Indirectly, it also improves the high-frequency behavior, as the shape-induced anisotropy field increases with the thickness of the magnetic film [18], [19].

The behavior observed for $Q$ can be understood as follows. Since the substrate and dielectric losses are negligible, the quality factor can be approximated as $Q=\omega L / R$, where the resistance per unit length $R$ accounts for both conductor loss and dissipation in the magnetic core. While the increase in $L$ (up to the FMR frequency) results in an improvement of $Q$, eddy currents induced in the magnetic core lead to an increase in $R$, which becomes more significant at higher frequencies. The quality factor is thus enhanced by a smaller factor than the inductance per unit length, and exhibits a smaller bandwidth in the frequency domain.

The inductance increase observed in these devices can be further improved by using a thicker magnetic film, as long as the thickness is kept small enough to limit the flow of eddy currents. Further increase of the ferromagnetic film thickness, however, would then come at the cost of large energy dissipation (low $Q$ ) and a sharp drop of the inductance with frequency [8], [9]. Reducing the thickness of the isolating oxide layers, on the other hand, would also lead to a larger inductance enhancement factor by increasing the effective cross section of the high-permeability ferromagnetic core. 


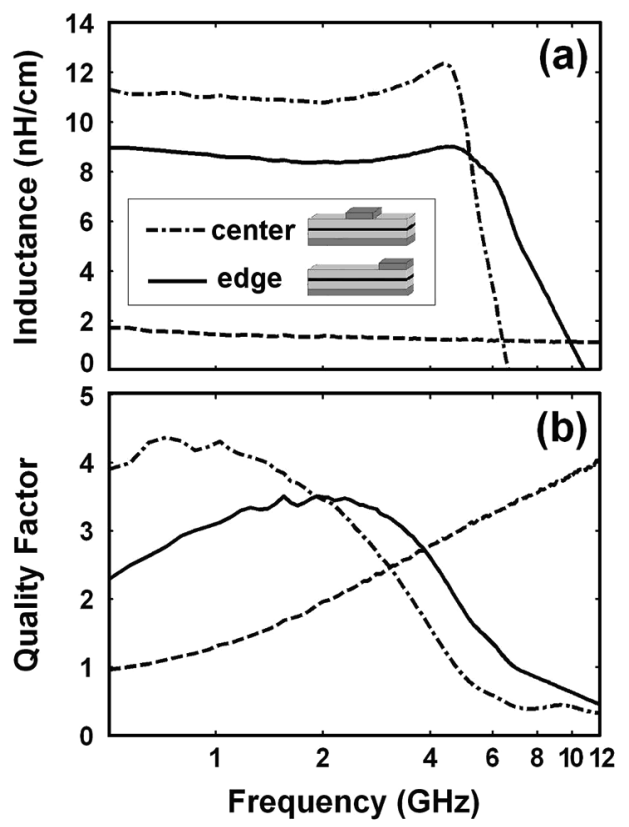

Fig. 4. (a) Inductance per unit length and (b) quality factor enhancement in microstrip transmission lines with a magnetic $\mathrm{Co}-\mathrm{Ta}-\mathrm{Zr}$ core, illustrating the effect of the signal line position. The signal line width is $20 \mu \mathrm{m}$ in this case, and the dashed lines represent a control device with no magnetic core. The larger shape-induced anisotropy field near the magnetic stripe edges can be used to increase the upper frequency limit (bandwidth) of the devices.

\section{EFFECT OF SignAl Line Position}

We next consider the effect of the signal line position on the FMR frequency. In order to more effectively probe the high demagnetizing field near the stripe edges, we use a narrower signal line in this case. Fig. 4 depicts the inductance and quality factors of microstrip lines with 20- $\mu \mathrm{m}$-wide signal lines, comparing devices with and without a $\mathrm{Co}-\mathrm{Ta}-\mathrm{Zr}$ magnetic core. Both cases of signal lines placed at the center and at the edge of the magnetic core are shown in this figure.

Looking at the centered line, firstly it can be seen that the inductance enhancement in this device (a factor of $\sim 8.5$ at $4 \mathrm{GHz}$ ) is slightly smaller than the case of the $50-\mu \mathrm{m}$-wide signal line shown in Fig. 3. Presumably, this is a result of the larger effect of fringing fields for the narrow line. Similar to the previous case, the inductance $L$ remains higher than that of the control lines up to $\sim 6 \mathrm{GHz}$.

Consistent with the expectation of a higher overall anisotropy field, the microstrip with a $20-\mu \mathrm{m}$-wide signal line placed at the edge of the magnetic stripe exhibits an improved frequency behavior, and the inductance enhancement in this case is maintained up to $\sim 10 \mathrm{GHz}$. The larger anisotropy, however, also leads to a smaller value for the relative permeability, which is given by $\left(M_{0}+H_{a}\right) / H_{a}$ at low frequencies. Here, $M_{0}$ is the saturation magnetization and $H_{a}$ is the magnetic anisotropy field, accounting for both internal and shape-induced contributions. This translates into a smaller increase of inductance than in the device with a centered signal line. Nevertheless, inductance enhancement by a factor of $\sim 6$ is still realized at $4 \mathrm{GHz}$ in this case.

When compared to the centered case, the smaller permeability also reduces the characteristic impedance of the line.

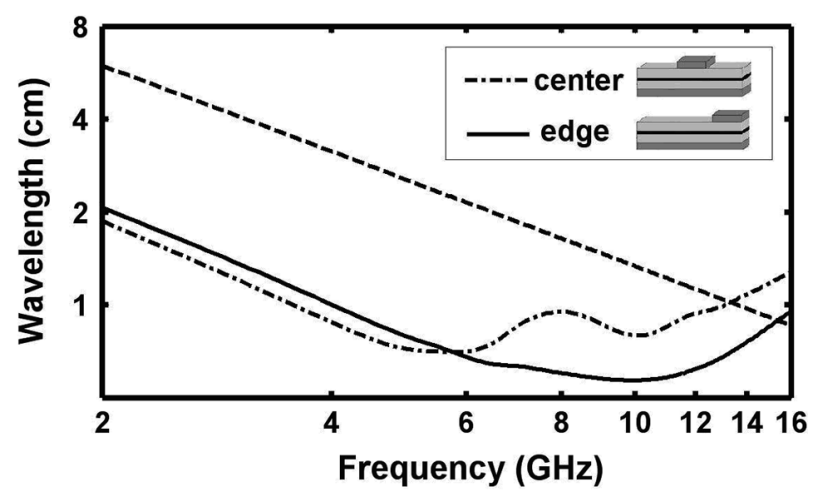

Fig. 5. Wavelength reduction in microstrip transmission lines due to the integration of a magnetic $\mathrm{Co}-\mathrm{Ta}-\mathrm{Zr}$ core. The dashed lines represent a control device with no magnetic core.

However, in cases where a given value (e.g., $50 \Omega$ ) is needed for the characteristic impedance, this can be compensated by reducing the signal line width correspondingly. A similar bandwidth improvement is seen in the quality factor, which remains higher than the control line values up to $\sim 4 \mathrm{GHz}$ for the signal line placed at the core edge, as compared to $\sim 3 \mathrm{GHz}$ for the centered line. Exploiting the nonuniform distribution of the shape-induced anisotropy is thus a possible option for optimizing the high-frequency performance of magnetic RF passives.

The incorporation of the magnetic Co-Ta-Zr core, due to its high permeability, also leads to a shortening of the electromagnetic propagation wavelength on the line (given by $\lambda=2 \pi / \beta$ ). As shown in Fig. 5, the wavelength reduction in microstrips with both center and edge signal lines is maintained up to $>12 \mathrm{GHz}$. Due to the lower effective permeability, the wavelength reduction factor is somewhat smaller in the case of the signal line placed near the magnetic stripe edge, but extends to higher frequencies $(\sim 15 \mathrm{GHz})$ instead. This wavelength shortening can be particularly important for the size reduction of distributed microwave devices such as quarter-wavelength transformers and phase shifters.

\section{CONCLUSION}

Device characteristics of microstrip lines with $\mathrm{Co}-\mathrm{Ta}-\mathrm{Zr}$ films were shown to be significantly improved with respect to both nonmagnetic control devices and previous results with other metallic magnetic materials. Inductance of the transmission lines was increased by a factor of $\sim 11$ and remained constant up to $\sim 4.5 \mathrm{GHz}$. The nonuniform shape-induced anisotropy field profile in the patterned magnetic films was used to enhance the FMR frequency in the devices. A maximum inductance enhancement frequency of $\sim 10 \mathrm{GHz}$ was achieved in this manner.

\section{ACKNOWLEDGMENT}

The authors would like to thank the integrated circuit processing and measurement staff of the Delft Institute of Microsystems and Nanoelectronics (DIMES), in particular Atef Akhnoukh and Hugo Schellevis, for their support. This 
work was supported by the technology foundation STW of the Netherlands. The work at Stanford was supported in part by the Nanoelectronics Research Initiative (NRI) through the Western Institute of Nanoelectronics (WIN).

\section{REFERENCES}

[1] V. Korenivski and R. B. Dover, "Design of high frequency inductors based on magnetic films," IEEE Trans. Magn., vol. 34, pp. 1375-1377, 1998.

[2] M. Yamaguchi, K. Suezawa, K. I. Arai, Y. Takahashi, S. Kikuchi, Y. Shimada, W. D. Li, S. Tanabe, and K. Ito, "Microfabrication and characteristics of magnetic thin-film inductors in the ultrahigh frequency region," J. Appl. Phys., vol. 85, no. 11, pp. 7919-7922, 1999.

[3] A. M. Crawford, D. Gardner, and S. X. Wang, "High-frequency microinductors with amorphous magnetic ground planes," IEEE Trans. Magn., vol. 38, no. 5, pp. 3168-3170, Sep. 2002.

[4] Y. Zhuang, M. Vroubel, B. Rejaei, and J. N. Burghartz, "Ferromagnetic $\mathrm{RF}$ inductors and transformers in standard CMOS/BiCMOS," IEDM Tech. Dig., pp. 475-478, 2002.

[5] D. S. Gardner, G. Schrom, P. Hazucha, F. Paillet, T. Karnik, and S. Borkar, "Integrated on-chip inductors with magnetic films," IEEE Trans. Magn., vol. 43, no. 6, pp. 2615-2617, Jun. 2007.

[6] Y. Zhuang, M. Vroubel, B. Rejaei, and J. N. Burghartz, "Integrated RF inductors with micro-patterned NiFe core," Solid-State Electron., vol. 51, no. 3, pp. 405-413, 2007.

[7] N. Cramer, D. Lucic, D. K. Walker, R. E. Camley, and Z. Celinski, "Incorporation of ferromagnetic metallic films in planar transmission lines for microwave device applications," IEEE Trans. Magn., vol. 37, no. 4, pp. 2392-2394, Jul. 2001.

[8] Y. Zhuang, M. Vroubel, B. Rejaei, E. Boellaard, and J. N. Burghartz, "Investigation of microstrips with NiFe magnetic thin film (I): Experiment," Trans. Magn. Soc. Japan, vol. 2, no. 5, pp. 367-370, 2002.

[9] M. Vroubel, Y. Zhuang, B. Rejaei, and J. N. Burghartz, "Investigation of microstrips with NiFe magnetic thin film (II): Modeling," Trans. Magn. Soc. Japan, vol. 2, no. 5, pp. 371-376, 2002.

[10] P. K. Amiri, B. Rejaei, Y. Zhuang, M. Vroubel, and J. N. Burghartz, "Ferromagnetic thin films for loss reduction in on-chip transmission lines," IEEE Trans. Magn., vol. 43, no. 6, pp. 2630-2632, Jun. 2007.

[11] B. Kuanr, D. L. Marvin, T. M. Christensen, R. E. Camley, and Z. Celinski, "High-frequency magnetic microstrip local bandpass filters," Appl. Phys. Lett., vol. 87, p. 222506, 2005.
[12] B. Kuanr, I. R. Harward, D. L. Marvin, T. Fal, R. E. Camley, D. L. Mills, and Z. Celinski, "High-frequency signal processing using ferromagnetic metals," IEEE Trans. Magn., vol. 41, no. 10, pp. 3538-3543, Oct. 2005.

[13] M. Vroubel, Y. Zhuang, B. Rejaei, and J. N. Burghartz, "Integrated tunable magnetic RF inductor,' IEEE Electron Dev. Lett., vol. 25, no. 12, pp. 787-789, Dec. 2004.

[14] P. K. Amiri, B. Rejaei, M. Vroubel, and Y. Zhuang, "Nonreciprocal spin wave spectroscopy of thin Ni-Fe stripes," Appl. Phys. Lett., vol. 91, no. 6, p. 062502, 2007.

[15] J. W. Wang, S. D. Yoon, V. G. Harris, C. Vittoria, and N. X. Sun, "Integrated metal magnetic film coupled line circulators for monolithic microwave integrated circuits," Electron. Lett., vol. 43, no. 5, pp. 49-50, 2007.

[16] S. X. Wang, N. X. Sun, M. Yamaguchi, and S. Yabukami, "Sandwich films: Properties of a new soft magnetic material," Nature, vol. 407, pp. 150-151, 2000.

[17] L. Li, D. W. Lee, M. Mao, T. Schneider, R. Bubber, K.-P. Hwang, Y. Min, and S. X. Wang, "High-frequency responses of granular CoFeHfO and amorphous CoZrTa magnetic materials," J. Appl. Phys., vol. 101, no. 12, p. 123912, 2007.

[18] G. Perrin, J. C. Peuzin, and O. Acher, "Control of the resonance frequency of soft ferromagnetic amorphous thin films by strip patterning," J. Appl. Phys., vol. 81, no. 8, pp. 5166-5168, 1997.

[19] M. Vroubel, Y. Zhuang, B. Rejaei, J. N. Burghartz, A. M. Crawford, and S. X. Wang, "Calculation of shape anisotropy for micropatterned thin Fe-Ni films for on-chip RF applications," IEEE Trans. Magn., vol. 40, no. 4, pp. 2835-2837, Jul. 2004.

[20] R. I. Joseph and E. Schlömann, "Demagnetizing field in nonellipsoidal bodies," J. Appl. Phys., vol. 36, no. 5, pp. 1579-1593, 1965.

[21] X. H. Huang and M. Pardavi-Horvath, "Local demagnetizing tensor calculation for arbitrary non-ellipsoidal bodies," IEEE Trans. Magn., vol. 32, no. 5, pp. 4180-4182, Sep. 1996.

[22] P. K. Amiri, B. Rejaei, M. Vroubel, Y. Zhuang, and J. N. Burghartz, "Experimental determination of the nonuniform shape-induced anisotropy field in thin Ni-Fe films," IEEE Trans. Magn., vol. 43, no. 5, pp. 1880-1883, May 2007.

Manuscript received March 03, 2008. Current version published December 17, 2008. Corresponding author P. Khalili Amiri : (e-mail: p.khalili@dimes. tudelft.nl). 